\title{
Recent Advances in Management of Postoperative Vomiting for Laparoscopic Bariatric Surgeries
}

\author{
Alaa Abbass Moustafa, Mohammed Abd El Monem Marzouk, Mina Ezzat Almaz Nesem * \\ General Surgery Department, Faculty of Medicine, Ain Shams University \\ * Corresponding Author: Mina Ezzat Almaz Nesem, E-mail: minaezat91@ gmail.com
}

\begin{abstract}
Background and Objectives: obesity continues to be a leading public health concern associated with many comorbidities that significantly decrease life expectancy. Surgery remains the only effective treatment modality for morbid obesity, resulting in long-term weight loss and sustained improvement in weight-related comorbidities. Vomiting is considered as a possible postoperative complication in all bariatric procedures. Anastomotic leak is one of the most serious complications following bariatric laparoscopic Roux-en-Y gastric bypass (LRYGB), and associated with high morbidity rates and prolonged hospital stay. Timely management is of utmost importance for the clinical outcome. This study evaluated the approach to suspected leakage in a high-volume bariatric surgery unit.

Patients and Methods: the study has been conducted on 100 Patients with morbid obesity. Observational checklist including: Age at the time of operation, body mass index (BMI), preoperative gastroesophageal reflux disease (GERD) and newly developed postoperative upper gastrointestinal symptoms. Invasive manoeuvres as gastrographin swallow and meal, virtual gastroscopy and upper gastroIntestinal endoscopy were done upon patients who developed post-operative vomiting after LBS for two months. Go to:

Results: 100 Patients who underwent LBS included $85 \%$ women, $42 \pm 10$ years old, body mass index 43,8 \pm $5,4 \mathrm{~kg} / \mathrm{m} 2$, sleeve gastrectomy $71 \%$, minigastric bypass surgery $24 \%$, Roux-en-Y gastric bypass $5 \%$. During the first 48 post-operative hours, $39 \%$ of patients developed postoperative vomiting which was controlled with medical treatment (Aprepitant). 4\% of them suffered from recurrent episodes of vomiting over a period of two months were surgically complicated (stenosis, kinking, or obstruction). Endoscopic treatment was successful for 3 of the 4 patients (75\%) after balloon dilatation or insertion of a stent. One of the 4 patients (25\%) required conversion to Roux-en-Y gastric bypass.

Conclusion: post-operative vomiting after Laparoscopic Bariatric Surgeries (LBS) is a common complication which responds well to centrally acting antiemetic (Aprepitant) during the first 48 post-operative hours. While invasive maneuvers (Balloon dilatation or insertion of a stent) or even surgical correction might be needed.
\end{abstract}

Keywords: Laparoscopic Bariatric Surgeries, Obesity, Vomiting, Aprepitant.

\section{INTRODUCTION}

Obesity continues to be a leading public health concern associated with many comorbidities that significantly decrease life expectancy. Surgery remains the only effective treatment modality for morbid obesity, resulting in long-term weight loss and sustained improvement in weight-related comorbidities ${ }^{(1)}$. Bariatric operations are either: restrictive, limiting the amount of food ingested (e.g.; adjustable gastric banding and sleeve gastrectomy), malabsorptive, limiting the amount of nutrients absorbed (e.g.; Biliopancreatic diversion), or a combination of both (e.g.; Rouxen-Y Gastric bypass surgery) ${ }^{(2)}$. Bariatric surgery had tremendous growth since its initial sporadic introduction in 1954 with a more than 20-fold increase in the number of procedures performed over the last decade ${ }^{(3)}$. Individuals with $\mathrm{BMI} \geq 40$ $\mathrm{kg} / \mathrm{m}^{2}$ and those with BMI > $35 \mathrm{~kg} / \mathrm{m}^{2}$ with obesity-related comorbidities who failed diet, exercise, and drug therapy, should be considered for bariatric surgery ${ }^{(4)}$. The rising prevalence of overweight and obesity in several countries has been described as a global pandemic. Obesity can be considered like the driving force towards the pre-mature deaths. It increases the likehood for the development of diabetes, hypertension and NASH. The American Heart Association identified obesity as an independent risk factor for the development of coronary heart disease. In order to minimize post-surgical cardiovascular risk, surgical weight loss may become a more frequently utilized option to address obesity. ${ }^{(\mathbf{1})}$. In the malabsorptive type of surgery as Rou-en-Y gastric bypass, there is surgical connection (anastomosis) between the stomach and bowel, or between two parts of the bowel. The surgeon attempts to create a water-tight connection by connecting the two organs with either staples or sutures, either of which actually makes a hole in the bowel wall. The surgeon will rely on the body's natural healing abilities and its ability to create a seal, like a self-sealing tire, to succeed with the surgery. If that seal fails to form for any reason, fluid from within the gastrointestinal tract can leak into the sterile abdominal cavity and give rise to infection and abscess formation. Leakage of an anastomosis can occur in about $2 \%$ of Roux-en-Y gastric bypass and less than $1 \%$ in mini gastric bypass ${ }^{(5)}$. As the anastomosis heals, it forms scar tissue, which 
naturally tends to shrink ("contract") over time, making the opening smaller. This is called a "stricture". Usually, the passage of food through an anastomosis will keep it stretched open, but if the inflammation and healing process outpaces the stretching process, scarring may make the opening so small that even liquids can no longer pass through it ${ }^{(6)}$. Vomiting is considered as a possible postoperative complication in all bariatric procedures because the focus of the operation is related to the stomach, so during the recovery process, it is common that there will be some discomfort in this area. Its causes may be medical (e.g. side effect of anaesthetics and stress gastritis) or technical (e.g.; blood clot, kinking, obstruction and adhesions) ${ }^{(7)}$.

\section{AIM OF THE WORK}

The aim of this study is to evaluate the percentage of post-operative vomiting occurrence after LBS for two months, its causes either medical or technical during operation with investigations and ways of management.

\section{PATIENTS AND METHODS}

This is a retrospective clinical trial included 100 patients. All patients underwent Laparoscopic Bariatric Surgeries over a 6 months period in Ain Shams University Hospitals during the period from December 2017 to June 2018. Preoperative evaluation followed the same standard protocol and included an accurate personal, medical and surgical history, complete endocrinal workup, psychological testing, and counseling by a dietician and followed a low caloric diet in arrange of one to three weeks according to BMI. All patients underwent a standard evaluation preoperatively concerning H-pylori test using noninvasive H-pylori antigen rapid test in patients' stool who was positive for H-pylori received the triple therapy treatment: Proton Pump Inhibitor ( Pantoprazole $40 \mathrm{mg}$ twice daily ), amoxicillin (1 g twice daily), and clarithromycin (500 mg twice daily) for 2 weeks preoperatively. Blood tests were requested in the form of complete blood picture, fasting blood sugar, lipid profile (cholesterol, LDL, HDL, triglycerides) Clinical chemistries (serum albumin, ALT, AST, GGT, Urea, Creatinine), pregnancy test for female patients and prothrombin time and concentration. Abdominal ultrasonography, chest X-ray were performed preoperatively. Thromboembolic prophylaxis with subcutaneous low molecular weight heparin was administered on the evening prior to surgery and continued daily from the first post-operative day till one week pos-operatively. Comorbidities that increase peri-operative risk as hypertension and diabetes were controlled before surgery. Informed written consent was obtained with explanation of the possible complications that could occur in the peri-operative period, which was specifically addressed. All patients were informed about the nature of the research, and each patient understood and agreed to the procedure after approval obtained from the Research Ethical Committee of the department of General surgery, Faculty of Medicine, Ain Shams University. Inclusion Criteria: The subjects were considered appropriate candidates for the present study if they were: Morbidly obese patients between $18-55$ years old willing and able to give consent, patients of both genders (male and female), patients with BMI (> $\left.40 \mathrm{~kg} / \mathrm{m}^{2}\right)$ or BMI $\left(35-39.9 \mathrm{~kg} / \mathrm{m}^{2}\right)$ with comorbidites like hypertension and diabetes, patients are generally fit for anesthesia and surgery. all appropriate non-surgical measures had been tried and failed to achieve or maintain adequate, clinically beneficial weight loss for at least six months. Exclusion Criteria: Extremities of age, body Mass Index above $55 \mathrm{~kg} \backslash \mathrm{m}^{2}$, pregnancy or lactation at screening for surgery, previous malabsorptive or restrictive procedures performed for the treatment of obesity. Postoperative regimen: Patients are encouraged to move out of bed few hours after surgery, anticoagulation DVT prophylaxis (enoxaparin 40 IU/day S.C) was given till one week post-operative. We start administration of IV PPI from first post-operative day, which is continued orally after patients start oral feeding. Data Collection: All patients were followed up for early post-operative complications like bleeding, leakage, vomiting and superficial or deep infections. The primary study objective was to evaluate the percentage of post-operative vomiting occurrence after LBS for two months, its causes with investigations and ways of management. Statistical Analysis: The software used for data entry was Microsoft Excel and for data analysis was SPSS statistical package- version 22. After cleaning of the data, descriptive analyses were performed in form of frequency and percentages for qualitative variables and mean \pm standard deviation for quantitative one. 


\section{RESULTS}

Table (1): Sex distribution

\begin{tabular}{|c|c|c|}
\hline Female & Male & Total \\
\hline 85 & 15 & 100 \\
\hline
\end{tabular}

There were 85 females and 15 males.

Table (2): Age distribution between males and females.

\begin{tabular}{|l|c|c|c|}
\hline \multicolumn{1}{|c|}{ Age } & Female & male & Total \\
\hline $\begin{array}{l}\text { Range of age } \\
\text { (Years) }\end{array}$ & $18-52$ & $26-55$ & $18-55$ \\
\hline Mean(years) & 37.3 & 41.8 & 37.9 \\
\hline
\end{tabular}

The age of the patients ranged between 18 and 55 years old with mean of 37.9 years old for all patients. With males ranging between 26 and 55 years old with mean of 41.8 years and females ranging between 18 and 52 years with a mean of 37.3 years as shown in table (2).

Table (3): BMI distribution between males and females.

\begin{tabular}{|l|c|c|c|}
\hline \multicolumn{1}{|c|}{ BMI } & Female & Male & Total \\
\hline Range $\left(\mathbf{K g} / \mathbf{m}^{2}\right)$ & $39-55$ & $37.9-53.2$ & $37.9-55$ \\
\hline Median $\left(\mathbf{K g} / \mathbf{m}^{2}\right)$ & 48.3 & 46.9 & 47.8 \\
\hline
\end{tabular}

The body mass index of those morbidly obese patients included in this study was ranging from 37.9 to $55 \mathrm{~kg} / \mathrm{m}^{2}$. (Median BMI for female was $48.3 \mathrm{~kg} / \mathrm{m}^{2}$ and for male was $46.9 \mathrm{~kg} / \mathrm{m}^{2}$ ) with a median for all patients is $47.8 \mathrm{~kg} / \mathrm{m}^{2}$ (table 3 ).

Table (4): Special habits distribution

\begin{tabular}{|l|c|c|}
\hline & Non-smokers & Smokers \\
\hline No. of patients & 86 & 14 \\
\hline Percentage of patients & $86 \%$ & $14 \%$ \\
\hline
\end{tabular}

The majority of patients were non-smokers (86\%). While percentage of smoker patients was (14\%) as shown in table (4).

Table (5): Pre-operative H. Pylori infection distribution

\begin{tabular}{|l|c|c|}
\hline & Positive & Negative \\
\hline No. of patients & 26 & 74 \\
\hline Percentage of patients & $\mathbf{2 6 \%}$ & $\mathbf{7 4 \%}$ \\
\hline
\end{tabular}

Searching for H-pylori antigen in stool before surgery. (74\%) rendered negative where $(26 \%)$ were marked positive by the test (table 5).

\section{DISCUSSION}

Obesity is considered as a global pandemic in several countries. It increases the likehood for the development of diabetes mellitus, hypertension, ischemic heart disease and NASH. Individuals with BMI $\geq 40 \mathrm{~kg} / \mathrm{m}^{2}$ and those with BMI > 35 $\mathrm{kg} / \mathrm{m}^{2}$ with obesity-related comorbidities who failed diet, exercise, and drug therapy, should be considered for bariatric surgery (1). Bariatric operations are either: restrictive, limiting the amount of food ingested (e.g.; adjustable gastric banding and sleeve gastrectomy), malabsorptive, limiting the amount of nutrients absorbed (e.g.; Biliopancreatic diversion), or a combination of both (e.g.; Roux-en-Y Gastric bypass surgery) ${ }^{(2)}$. Vomiting is a common side effect after LBS. Postoperative vomiting is often triggered by different things, such as anxiety, illness, perioperative stimuli, anesthesia, drug interactions, marginal ulceration, staple line failure, stenosis/obstruction, gastro-gastric fistula, gastrojejunostomy anastomotic stricture, pouch dilation, and pouch gastritis ${ }^{(8)}$. In our study, 100 patients who underwent LBS were included in this retrospective clinical trial over a 6 months period to evaluate the percentage of post-operative vomiting occurrence after surgery for two months of follow up. The majority of patients are females in the late thirties. $26 \%$ were marked positive after searching for $\mathrm{H}$ pylori antigen in stool. The study declared a high percentage of post-operative vomiting in 39\% of cases within the first 48 hours of operation. Most of them showed a significant improvement with the use of centrally acting antiemetic drug. $4 \%$ of patients suffered from persistent vomiting during the post-operative 2 months. They were investigated by virtual gastroscopy, UGIE and CT scan with contrast. Two cases suffered from moderate stenosis who underwent balloon dilatation. One case with obstruction was resolved with stent insertion. The last complicated case was managed surgically with conversion to Roux-en-Y gastric bypass. Several investigators commented on the anatomic and physiologic effects of LBS and postulated their effect on GERD. Using manometry, Braghetto et al. ${ }^{(9)}$ demonstrated that the reduced LES pressure after LSG could cause reflux symptoms and esophagitis. Klaus and Weiss ${ }^{(10)}$ reasoned that esophageal manometry might be a useful criterion in deciding whether to offer LBS. The lack of gastric compliance and 
emptying and the blunting of the angle of His inherent in LSG at 1 year were responsible for the increase GERD symptoms at 1 year after LBS. They postulated that an increase in gastric compliance and clearance after 3 years likely accounted for the resolution of GERD symptoms at 3 years. Finally, they also noted that barium swallow testing after 3 years revealed restoration of the angle of His, which might have accounted for the decrease in GERD. Carabotti et al. ${ }^{(11)}$ after a median follow-up of 13 months on 74 patients declared that an increase in the prevalence of upper GI symptoms was observed in $91.9 \%(68 / 74)$ of the patients complained of symptoms compared to $47.3 \% \quad(35 / 74)$ before surgery. Dyspeptic symptoms became the main complaint in $59.4 \%$ (44/74) of the patients. Fernandes et al. (12) analyzed the data of 613 patients undergoing UGE in a retrospective study, where all patients had an indication for bariatric surgery (BMI $>40 \mathrm{~kg} / \mathrm{m}^{2}$ or BMI $>35 \mathrm{~kg} / \mathrm{m}^{2}$ and one or more significant obesity-related comorbid conditions). $77.8 \%$ female, with mean age of $46.5 \pm 11.6$ years and mean BMI of $44.7 \pm 4.2 \mathrm{~kg} / \mathrm{m}^{2}$. UGIE presented at least one abnormal finding in 345 patients (56.3 $\%)$. Esophageal, gastric, and duodenal findings were reported in $22.2,41.8$, and $11.4 \%$ of patients, respectively. In accordance with his results, abnormal endoscopic findings had been reported in 10 to $90 \%$ of patients across studies. Nevertheless, excessive and unnecessary interventions had been brought forth by mild and insignificant endoscopic findings. This had led many authors to limit endoscopy to patients with symptoms of gastrointestinal disease. This may be inadequate, as several studies have reported pathologic endoscopic findings in asymptomatic patients. Other authors have abandoned routine UGE before surgery. There is a paucity of data regarding which endoscopic findings should delay, change, or contraindicate surgery. Fernandes et al. ${ }^{(13)}$ found gastric and duodenal ulcers to be the only significant predictors of postoperative complications. Almazeedi et al. ${ }^{(14)}$ compared H. pylori presence pre and post-operatively. They observed the prevalence of $\mathrm{H}$. pylori in the population undergoing bariatric surgery, and studied the impact of $\mathrm{H}$. pylori screening and eradication carried out by some surgeons. When observing the trend of $\mathrm{H}$. pylori infections before and after LBS, it was clear that its presence in symptomatic patients who underwent UGIE was high $(49.7 \%)$. Furthermore, since by the time of surgery $H$. pylori was only seen in $7.8 \%$ of patients, this indicates that pre-operative UGIE does help in eradicating the infection, especially in symptomatic patients. They revealed that most (93.6 \%) of the H. pylori-positive patients who took triple therapy became negative by the time of surgery. In contrast, more than half of the patients who were positive pre-operatively and did not take triple therapy remained positive at the time surgery performance.

\section{CONCLUSION}

From our study, we conclude the following: Our results showed that post-operative vomiting after LBS represented a significant complication, which occurs commonly after LBS. vomiting of medical causes responds well to centrally acting antiemetic and to a lesser extent, endoscopic and surgical interventions were required for technical problems. Further studies have to be applied to a larger number of patients to confirm results of the study.

\section{CONFLICTS OF INTEREST}

There are no conflicts of interest.

\section{REFERENCES}

1. Schauer PR, Kashyap SR, Wolski K, Brethauer SA, Kirwan JP, Pothier CE, Thomas S, Abood B, Nissen SE, Bhatt DL (2012): Bariatric surgery versus intensive medical therapy in obese patients with diabetes. New England Journal of Medicine, 366 (17): 1567-76.

\section{El-Banna A, Taweela NH, Gaber MB, El-} Din MM (2014): Medical management of patients with modified intestinal bypass: a new promising procedure for morbid obesity. Global Journal of Medical Research, 26: 331-239.

\section{White S, Brooks E, Jurikova L, Stubbs RS} (2005): Long-term outcomes after gastric bypass. Obesity Surgery, 15 (2): 155-63. 
4. Nguyen NT, Masoomi $\mathrm{H}$, Magno CP, Nguyen $\mathbf{X M}$, Laugenour $\mathbf{K}$, Lane $\mathbf{J}$ (2011): Trends in use of bariatric surgery, 2003- 2008. Journal of the American College of Surgeons, 213 (2): 261-6.

5. Sacks BC, Mattar SG, Qureshi FG, Eid GM, Collins JL, Barinas-Mitchell EJ, Schauer PR, Ramanathan RC (2006): Incidence of marginal ulcers and the use of absorbable anastomotic sutures in laparoscopic Roux-en-Y gastric bypass. Surgery for Obesity and Related Diseases, 2 (1): 11-6.

6. John S, Hoegerl C (2009): Nutritional deficiencies after gastric bypass surgery. The Journal of the American Osteopathic Association, 109 (11): 601-4.

7. Khoursheed M, Al-Bader I, Mohammad AI, Soliman MO, Dashti H (2007): Slippage after adjustable gastric banding according to the pars flaccida and the perigastric approach. Med Princ Pract., 16: 110-13.

8. Hawkins W, Maheswaran I (2016): The management of bariatric surgery complications. Surgery (Oxford), 34 (11): 563-567.
9. Braghetto I, Lanzarini E, Valladares $\mathbf{H}$, Valladares H, Molina JC (2010): Henrique Manometric changes of the lower esophageal sphincter after sleeve gastrectomy in obese patients. Obes Surg., 20: 357-62.

10. Klaus A, Weiss H (2008): Is preoperative manometry in restrictive bariatric procedures necessary? Obes Surg., 18: 1039-42.

11. Carabotti M, Silecchia G, Greco F et al. (2013): Impact of laparoscopic sleeve gastrectomy on upper gastrointestinal symptoms. Obes Surg., 23 (10): 1551-7.

12. Fernandes SR, Meireles LC, Carrilhoribeiro L, Velosa J (2016): The Role of Routine Upper Gastrointestinal Endoscopy before Bariatric Surgery. Obesity surgery, 26 (9): 2105-10.

13. Peromaa-Haavisto P, Victorzon M (2013): Is routine preoperative upper GI endoscopy needed prior to gastric bypass? Obes Surg., 23 (6): 736-9.

14. Almazeedi S, Al-Sabah S, Alshammari D et al. (2014): The Impact of Helicobacter pylori on the Complications of Laparoscopic Sleeve Gastrectomy. Obes Surg., 24: 412-415. 